\title{
Study on the Reinforcement Measures and Control Effect of the Surrounding Rock Stability Based on the Shield Tunneling Under Overpass Structure
}

\author{
Qian-cheng Fang ${ }^{1 *}$, Li Shang ${ }^{1}$, Yong-hui Shang ${ }^{2}$, Ying Zhao ${ }^{1}$ and Ning-yi OU ${ }^{3}$ \\ ${ }^{1}$ Institute of Architecture and Engineering, Huang Huai University, Zhumadian 463000, China \\ ${ }^{2}$ School of Civil Engineering, Central South University, Changsha 410083, China \\ ${ }^{3}$ Center of International Affairs, CEKII Civil Engineering Co .Ltd., Kowloon, Hong Kong
}

Received 11 November 2015; Accepted 1 April 2016

\begin{abstract}
To study the stability of surrounding rocks for shield tunneling under overpass structures and the safety of existing bridge structures, a practical example of the method was cited through a shield tunneling project under the overpass structure between $\mathrm{K} 1+110$ and $\mathrm{K} 1+700$ on Line 2 of Shenyang Subway, China. The sub-area reinforcement was proposed according to surrounding rock deformation characteristics during shield tunnel excavation. The bridge foundation (i.e., the clear spacing to the shield tunnel is less than $2 \mathrm{~m}$ ) was reinforced by steel support, the bridge foundation (the clear spacing is about $2 \sim 7 \mathrm{~m}$ ) used "jet grouting pile" reinforcement, whereas the bridge foundation (the clear spacing is greater than $7 \mathrm{~m}$ ) did not adopt any reinforcement measures for the moment. For this study, the mean value and material heterogeneity models were established to evaluate the reinforcement effect from several aspects, such as surrounding rock deformation, plastic zone development, and safety factor. The simulation results were consistent with those of field monitoring. After reinforcement, the maximum deformation values of the surrounding rock were reduced by $4.9 \%, 12.2 \%$, and $48.46 \%$, and the maximum values of surface subsidence were decreased by $5.6 \%, 72.2 \%$, and $88.64 \%$. By contrast, the overall safety factor was increased by $4.1 \%, 55.46 \%$, and $55.46 \%$. This study posited that this reinforcement method can be adopted to solve tunnel construction problems in engineering-geological conditions effectively. References for evaluating similar projects are provided.
\end{abstract}

Keywords: Shield, Overpass Structure, Surrounding Rock, Control Effect, Safety Factor

\section{Introduction}

With the rapid development of urban rail traffic, the subway has become a crucial part of urban underground space development in the 21st Century [1-3]. The construction of subway is often heavily restricted by the existing environment, with many subway lines constructed along urban roads. For example, Lines 4, 5, and 10 of the Beijing Subway, with a total underground length of $63.1 \mathrm{~km}$, go through more than ten foundations of overpass bridges on urban roads. At its closest, the distance to the bridge foundation is only $0.19 \mathrm{~m}$. Similar issues during subway construction have also been encountered in Shanghai, Guangzhou, Shenzhen and other cities [4]. How to ensure the safety of subway construction close to existing bridges has become an increasingly acute problem [5]. The disturbance to surrounding rock from a new tunnel construction inevitably causes deformation of and internal force on existing buildings. If the deformation exceeds the load-bearing capacity or the maximum allowable value, damage will be caused, which not only influences the normal use of the buildings but also threatens the safety of the new tunnel construction as well as its normal operation later [6-8]. Hence, under this situation, the scientific

* E-mail address: fangqiancheng314@126.com

ISSN: 1791-2377 @ 2016 Eastern Macedonia and Thrace Institute of Technology.

All rights reserved. reinforcement measure and key control technology are required to meet both safety construction of tunnels and normal use of existing buildings.

\section{State of the art}

Research on the effects of subway construction on adjacent bridge piers involves tunnel engineering, pile foundation engineering, soil mechanics, and other fields. Its complexity has led to the general weakness and slow progress of research work in this area. Loganathan [9] designed a centrifuge model, made 3 groups of centrifuge tests to estimate stratum deformation due to tunnel excavation in clay strata and its influence on adjacent pile foundations. The calculation formula was derived by Li Yongsheng [10], with the help of elastic mechanics and the theory of beams on elastic foundations, which described the effect on an adjacent pile body force and deflection by shield promoting. Using a three-dimensional finite element method, Ruan Linwang [11] researched deformation and stress caused to an adjacent pile by soft soil shield construction. Wang Zhansheng [12] systematically studied the shield closely through the pile foundation. He analyzed the effect on the pile foundation and the mechanism of influence factors, proposing a method for evaluating the effects on a neighboring single pile's internal force and the deformation by shield construction as well as the methods and procedures for shield tunneling going through pile foundations. He also 
emphasized the control of construction parameters during shield construction through the pile foundation and auxiliary construction measures. Burland [13] introduced the concept of protecting surface buildings with row pile structures in the extension of a subway line in the UK. Ye Shulin and Wang Yiji [14] described the protection of adjacent towers by isolation piles in Line 2 of the Shanghai Metro. Wang Wenbin [15] represented the protection of a clock tower by separation pillars in Line 2 Metro in Xi'an. The literatures [16-17] specified the reinforcement mechanism of the isolation pile. Nevertheless, at present, little research has been done on how to safely protect urban subway construction close to buildings. There remains a lack of systematic and universal norms and standards. Numerous previous studies focused on reinforcement measures and the influence of the adjacent building, while research on the control effect of strengthening is scarce.

The area between $\mathrm{K} 1+110$ and $\mathrm{K} 1+700$ on Line 2 metro in Shenyang crosses under an overpass bridge, the bridge pile of which is close to the tunnel entrance. The minimum horizontal distance from the bridge pile to the tunnel entrance is $0.4 \mathrm{~m}$, and the minimum vertical distance is $5.35 \mathrm{~m}$. In addition, the line's hydrogeological condition is very complex. Based on the research into engineeringgeological conditions and the surrounding environment, this article selects "steel supports" to control the influence on existing buildings adjacent to the new tunnel construction. Combined with measured data, a material mean value model and a material heterogeneity model were established. According to strength reduction and stochastic medium theory, the effect of reinforcement was assessed from the aspects of surrounding rock deformation, plastic zone development and safety factor. The research shows that this method can effectively solve difficulties encountered in tunnel construction when facing with this type of engineering-geological conditions, and also provide a new approach for assessing similar projects.

\section{Description of the Problem}

The 1st bidding zone of the line 2 of the Shenyang subway has a length of $1268.4 \mathrm{~m}$ and a design range from $\mathrm{K} 0+606.7$ to $\mathrm{K} 1+875.100$. The tunnel is constructed by the shield tunneling method (the machine is used by an earth pressure balance shield machine). It is a single hole and single line tunnel with a circular cross section. The space between the two lines is $13-50 \mathrm{~m}$. This section line goes as a "V" type slope in the longitudinal axis with a maximum slope of $30 \%$. The maximum buried depth of the tunnel is about $30.12 \mathrm{~m}$ (the thickness of covering soil is $24.12 \mathrm{~m}$ ), and the minimum buried depth is $14.98 \mathrm{~m}$ (the thickness of covering soil is $9.28 \mathrm{~m})$

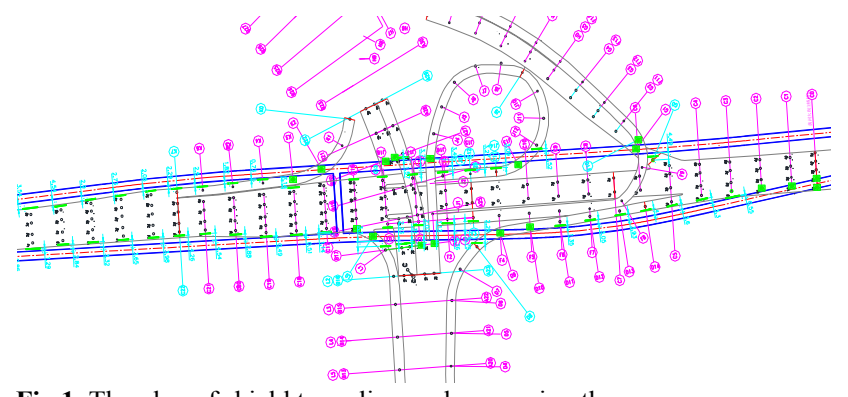

Fig.1. The plan of shield tunneling under-crossing the overpass
The way between $\mathrm{K} 1+110 \sim \mathrm{K} 1+700$ of the tunnel goes under an overpass (the structure of overpass was in good according to testing of the original design unit in 1999). The bridge foundation pile has a diameter of $2 \sim 2.5 \mathrm{~m}$, with a $13.3 \sim 17.2 \mathrm{~m}$ depth. Take the length as the classification standard: A (13m), B (14 14.7m), C (14 14.6m), D (13 16.9m), E (13 14.85m), F (11m), and G (13m).

Table 1. Distance from the bridge's foundation to the tunnel (the left line) entrance, self-elaboration

\begin{tabular}{ccccc}
\hline \multirow{2}{*}{ Number } & Pile & \multicolumn{3}{c}{ Distance from left line of tunnel (m) } \\
& & Left side & Right side & Ceiling \\
\hline 1 & C29 & - & 6.98 & 10.388 \\
2 & C25 & - & 8.3 & 6.592 \\
3 & C24 & - & 8.13 & 10.692 \\
5 & K2 & 0.40 & - & 10.736 \\
6 & K3 & - & 4.98 & 10.796 \\
7 & K4 & - & 6.13 & 10.896 \\
8 & K5 & - & 7.23 & 10.96 \\
9 & K6 & - & 7.91 & 10.992 \\
10 & K7 & - & 7.7 & 10.864 \\
11 & A27 & 6.97 & - & 9.664 \\
12 & J16 & - & 8.86 & 10.464 \\
13 & J15 & - & 8.67 & 10.376 \\
14 & J14 & - & 8.49 & 10.272 \\
15 & J12 & 6.92 & - & 10.076 \\
16 & G0 & - & 7.49 & 7.228 \\
17 & B20,1\# & 2.85 & - & 9.10 \\
18 & B20,2\# & 5.01 & - & 10.68 \\
19 & J3 & 2.77 & - & 10.356 \\
20 & J13 & - & 4.86 & 10.144 \\
21 & G1 & 0.42 & - & 7.964 \\
22 & F10 & 5.19 & - & 8.008 \\
\hline
\end{tabular}

Table 2.. Distance from the bridge's foundation to the tunnel (the right line) entrance, self-elaboration

\begin{tabular}{ccccc}
\hline \multirow{2}{*}{ Number } & \multirow{4}{*}{ Pile } & \multicolumn{3}{c}{ Distance from right line of tunnel (m) } \\
& & Left side & Right side & Ceiling \\
\cline { 3 - 5 } 1 & C29 & 5.53 & - & 10.39 \\
2 & C28 & 7.73 & - & 5.928 \\
3 & C27 & 8.05 & - & 6.243 \\
4 & C26 & 8.39 & - & 6.452 \\
5 & C25 & 8.72 & - & 6.696 \\
6 & C24 & 9.05 & - & 6.896 \\
7 & C23 & 9.38 & - & 7.172 \\
8 & C22 & 9.66 & - & 7.296 \\
9 & C21 & 9.94 & - & 11.124 \\
10 & C20 & 8.43 & - & 11.1 \\
11 & C19 & 6.04 & - & 10.056 \\
12 & C18 & 6.06 & - & 9.984 \\
13 & C17 & 6.44 & - & 9.912 \\
14 & B17,1\# & - & 7.50 & 9.148 \\
15 & B17,2\# & - & 6.93 & 9.036
\end{tabular}




\begin{tabular}{ccccc}
16 & D8 & - & 7.66 & 9.50 \\
17 & D11 & 6.73 & - & 10.344 \\
18 & D12 & 8.46 & - & 9.992 \\
19 & D13 & 9.32 & - & 9.356 \\
20 & D14 & 9.39 & - & 9.10 \\
21 & $\mathrm{C} 5$ & 9.00 & - & 8.344 \\
22 & $\mathrm{C} 4$ & 8.85 & - & 7.712 \\
23 & $\mathrm{C} 3$ & 7.37 & - & 6.676 \\
24 & $\mathrm{C} 16$ & 4.58 & - & 10.86 \\
25 & $\mathrm{~A} 24,1 \#$ & - & 5.07 & 10.104 \\
26 & $\mathrm{~A} 24,2 \#$ & - & 4.13 & 10.10 \\
27 & $\mathrm{D} 9$ & 0.91 & - & 10.444 \\
28 & $\mathrm{D} 10$ & 4.28 & - & 10.408 \\
29 & $\mathrm{C} 2$ & 4.92 & - & 6.052 \\
30 & $\mathrm{C} 1$ & 2.09 & - & 5.352 \\
31 & $\mathrm{C} 0-1$ & - & 1.33 & 7.444 \\
32 & $\mathrm{C} 0-2$ & 2.34 & - & 7.444 \\
\hline
\end{tabular}

The hydrogeological environment in the area is complicated. The various layers from top to bottom could be described as: miscellaneous fill, silty clay, coarse sand, sand, and mud gravel. The tunnel crosses mud gravel layer, the intensity of which is lower. The original soil had already been disturbed in the construction of the existing buildings, although the scope of the disturbance field was difficult to ascertain accurately. In shield tunnel construction, measures against precipitation were taken, which resulted in a disorderly distribution of the underground water system. The soil parameters are indicated in Table 3.

Table 3. Soil parameters (triaxial experiment), selfelaboration

\begin{tabular}{cccccc}
\hline Parameter & $\begin{array}{c}\text { Miscellane- } \\
\text { ous fill }\end{array}$ & $\begin{array}{c}\text { Silty } \\
\text { clay }\end{array}$ & $\begin{array}{c}\text { Coarse } \\
\text { sand }\end{array}$ & Sand & $\begin{array}{c}\text { Mud gravel } \\
\text { layer }\end{array}$ \\
\hline$\rho\left(\mathrm{KN} / \mathrm{m}^{3}\right)$ & 16.50 & 19.50 & 19.10 & 19.10 & 26.6 \\
$\mathrm{E}(\mathrm{Mpa})$ & 7.00 & 16.7 & 31.02 & 31.89 & 18.84 \\
$\begin{array}{c}\text { Poisson's } \\
\text { ratio } \delta\end{array}$ & 0.35 & 0.30 & 0.30 & 0.28 & 0.30 \\
$\begin{array}{c}\text { Cohesion } \\
\text { (Kpa) } \\
\text { friction }\end{array}$ & 10 & 32 & 3.00 & 2.00 & 2.00 \\
$\begin{array}{c}\text { angle }(\varphi) \\
\text { Depth }(\mathrm{m})\end{array}$ & 1.5 & 9.0 & 4.0 & 7.5 & $>38$ \\
\hline
\end{tabular}

\section{Methodology}

\subsection{Division of Stratum Deformation}

Based on references [10], [18] and [19], stratum deformation is divided into 2 areas caused by shield construction (Fig.2).

(1) The bridge pile foundations outside of areas I and II are not considered protective measures, only to strengthen the monitoring during construction.

(2) The method of isolation piles were taken between the shield construction and the pile foundation in part I and II of the areas as the figure shows, where their distances are more than $2 \mathrm{~m}$ (that is, after the shield construction, based on the size of the settlement monitoring results to determine whether take jet grouting pile or not).
(3) In the area where the clear spacing between the shield structure and pile is less than $2 \mathrm{~m}$, the bridge foundations were reinforced by steel supports.

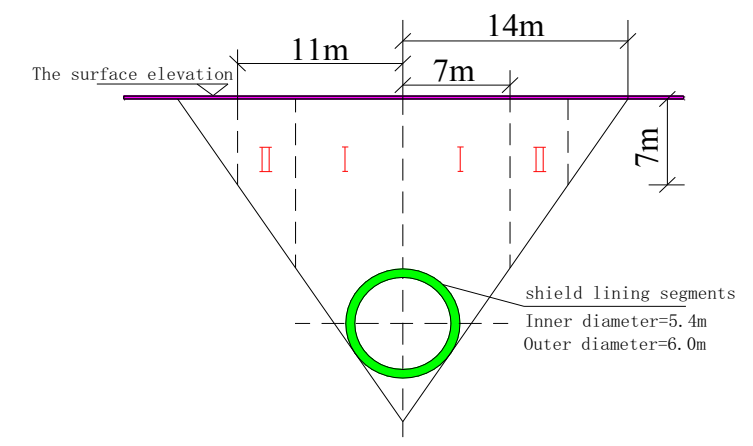

Fig.2. Deformation area caused by shield construction, self-elaboration

\subsection{Optimal Selection of Reinforcement Measures}

The original reinforcement of the bridge pier is intensive. The spacing of vertical main bar and stirrups is $10 \mathrm{~cm}$. If a Ф32 piece of steel in the pier is taken as a reinforced concrete corbel, most of the pier's main bars will be destroyed, the ability of the pier to withstand the upper load will be greatly weakened and the bridge structure will be unsafe. Furthermore, when the bridge pier settlement is stabilized, the pier could not recover to its original state if the jack and concrete corbel were removed. Thus, according to the actual situation of the pier, using steel support rather than the method of underpinning support can effectively avoid the destruction on the original structure of the pier.

The main technologies of shape steel support are: (i) the steel beam was constructed as the foundation; (ii) the steel beam was gently installed in a steel cushion, welding was used in the steel pipe support area in order to enhance stability of force; (iii) the upper pipe support was installed, which was manufactured in a factory and then transported to the site for installation; (iv) using the synchronous jacking technique make the steel pipe supports evenly force. Each beam on the top of the steel support touched the bridge tight and closely. When the shield machine passed through the foundation of the bridge and the settlement reached the warning value $5 \mathrm{~mm}$, the bridge would be restored to its original position and fixed by the jack. Figure 3 shows the construction sites.

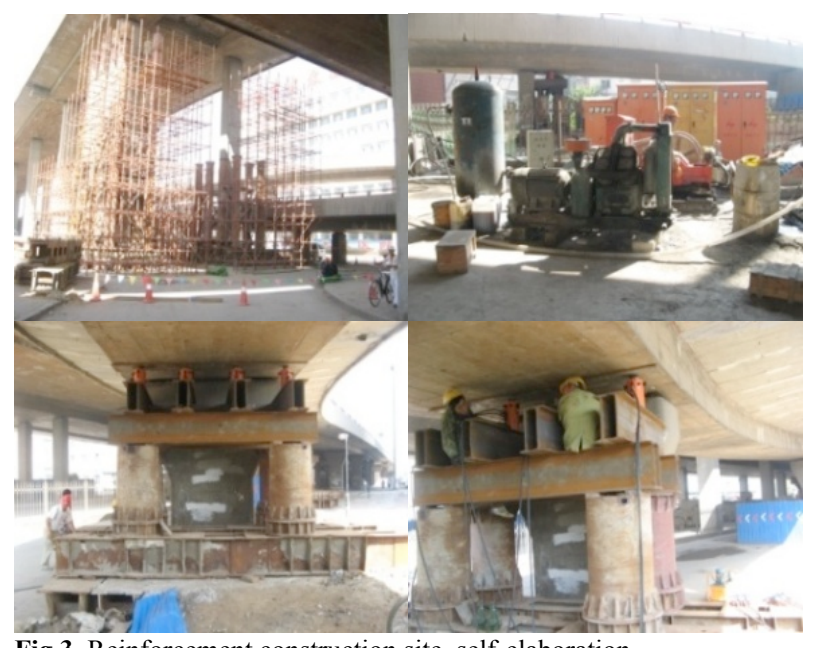

Fig.3. Reinforcement construction site, self-elaboration 


\subsection{Numerical Simulation Analysis}

Existing evaluation work on the strengthening control effect continues to suffer from a lack of standards and specifications. This article conducts its evaluation through numerical simulation and on-site monitoring.

According to the spatial positions of pile foundations and the tunnel in the research area, 3 representative conditions were selected (principles: abutment distance closer to the unfavorable tunnel face section) in order to analyze the specific details in Table 4. According to Gunn [2], recommendations exist for length and width (4D and 5D respectively, where $\mathrm{D}=6.28 \mathrm{~m}$, the diameter of tunnel excavation hole).

Table 4. Analysis of conditions, self-elaboration

\begin{tabular}{|c|c|c|c|c|c|c|}
\hline \multirow{2}{*}{ condition } & \multirow{2}{*}{ Pile } & \multicolumn{3}{|c|}{ Bridge foundation - tunnel location(m) } & \multirow{2}{*}{ Tunnel buried depth } & \multirow{2}{*}{ Reinforcement } \\
\hline & & Left & Right & Ceiling & & \\
\hline 1 & $\mathrm{C} 21$ & 9.94 & - & 11.12 & 27.62 & Isolation pile \\
\hline 2 & G1 & 0.42 & - & 7.96 & 27.59 & \\
\hline \multirow{2}{*}{3} & $\mathrm{C} 0-1$ & - & 1.33 & 7.44 & \multirow{2}{*}{27.63} & Steel reinforcement \\
\hline & $\mathrm{C} 0-2$ & 2.34 & - & 7.44 & & \\
\hline
\end{tabular}

First, a mean isotropic material model was established by ABAQUS to analyze the plastic zone of surrounding rock deformation and its development in different conditions after reinforcement. Then, with the help of RFPA-2d, a material anisotropy heterogeneous model was established. The analysis considers constitutive model with residual strength after elastic damages and the failed elements are treated with reduced properties. The progressive failure characteristics of the surrounding rock under reduction degradation were analyzed before and after reinforcement, and the overall safety factor was ascertained.

\subsection{Results of Numerical Simulation Analysis}

A model is established by ABAQUS: a CPE4R unit was adopted by the pile, lining and grouting (a 4-node plane strain reduced integral entity unit), and the soil was represented using a CPE4P unit (a 4-node plane strain pore pressure entity unit). The excavation process of tunnel was simulated by the birth-death element method, properties of soil mass were adopted by the Duncan-Chang constitutive Model, and the others were adopted by linear-elastic constitutive model. The required reference data of soil is detailed in Table 3, and the results are shown in Fig.4 and Fig.5.

The calculation results showed that deformation of the surrounding rock was mainly concentrated in the mouth of the cave and below, and a "tank" appeared in the mouth of the cave after tunnel excavation. The size of reduction was small in condition 1, but was larger in conditions 2 and 3 after reinforcement measures were taken, because the bridge foundations were farther from the excavation section surface of tunnel in condition 1, the effect is less serious than that in condition 2 and 3.

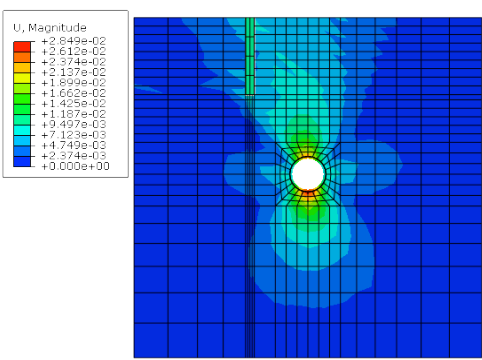

(1)condition 1

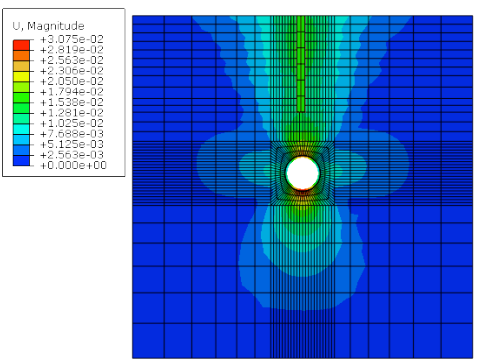

(2)condition 2

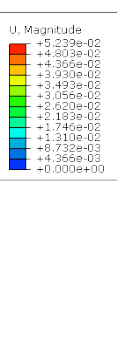

(a) Surrounding rock deformation nephogram(before strengthening)

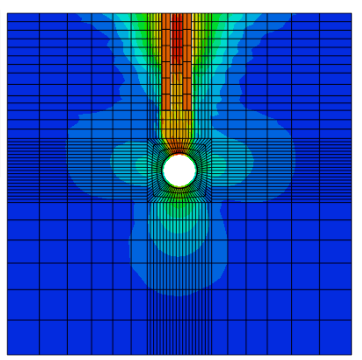

(3)condition 3

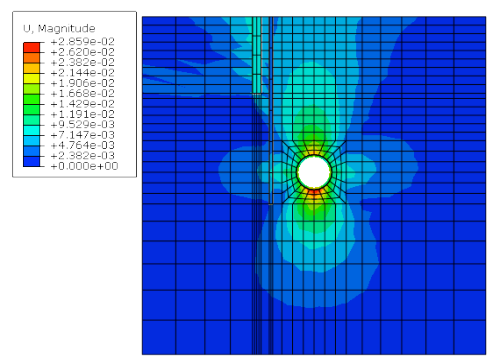

(1)condition 1

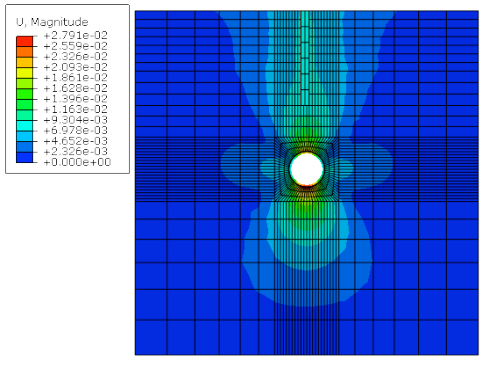

(2)condition 2

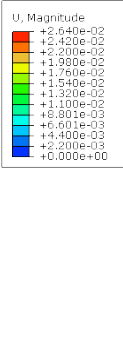

(b)Surrounding rock deformation nephogram(after strengthening)

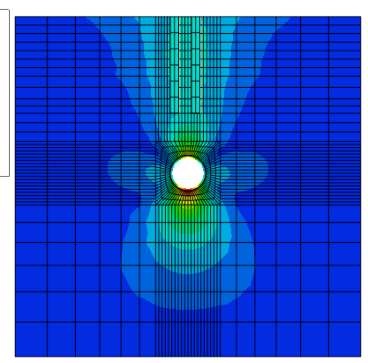

(3)condition 3 


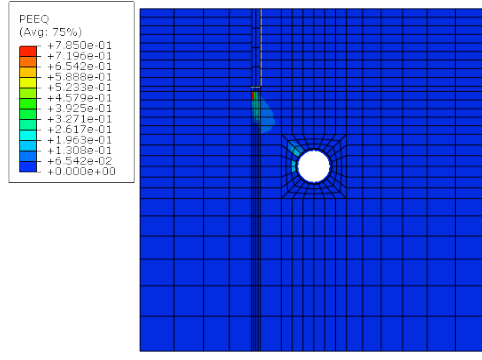

(1)condition 1

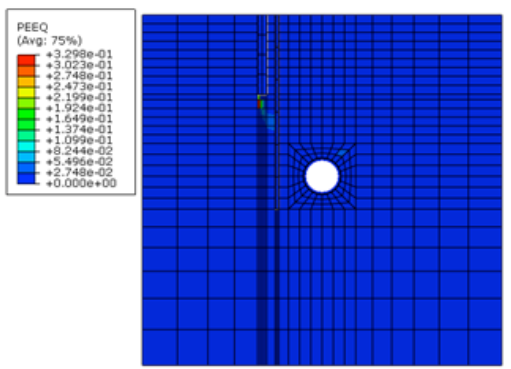

(1)condition 1

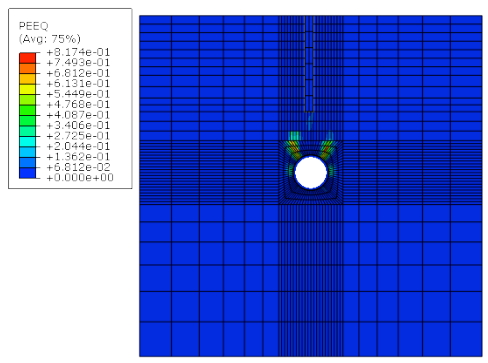

(2)condition 2

(c)Plastic strain contours(before strengthening)
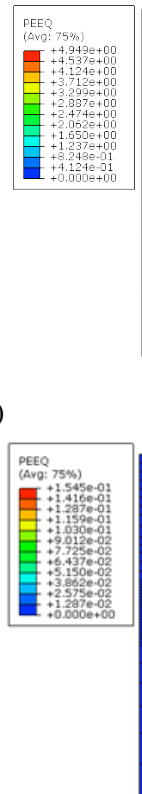

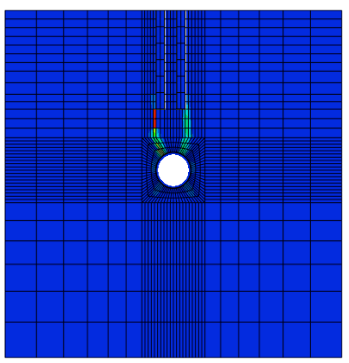

(3)condition 3 (2)condition 2

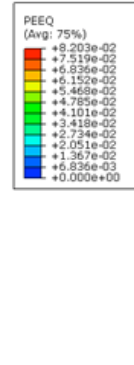

(d)Plastic strain contours(after strengthening)

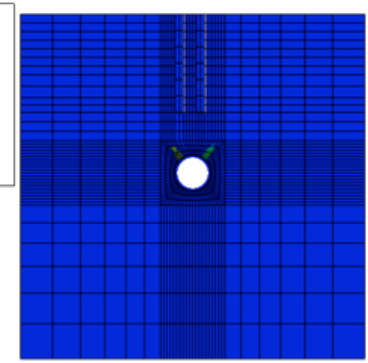

(3)condition 3

Fig.4. Nephogram of calculation results, self-elaboration

The maximum deformation of the surrounding rock under working conditions $1 \sim 3$ were $28.4 \mathrm{~mm}, 30.75 \mathrm{~mm}$ and $52.39 \mathrm{~mm}$ before reinforcement, falling to $27 \mathrm{~mm}$ after reinforcement and hence reduced by $4.9 \%, 12.2 \%$ and $48.46 \%$ respectively. The surface subsidence under working conditions $1 \sim 3$ were $5.3 \mathrm{~mm}, 18 \mathrm{~mm}$ and $44 \mathrm{~mm}$ before reinforcement, essentially falling to about $5 \mathrm{~mm}$ after reinforcement and hence reduced by $5.6 \%, 72.2 \%$ and $88.64 \%$ respectively.

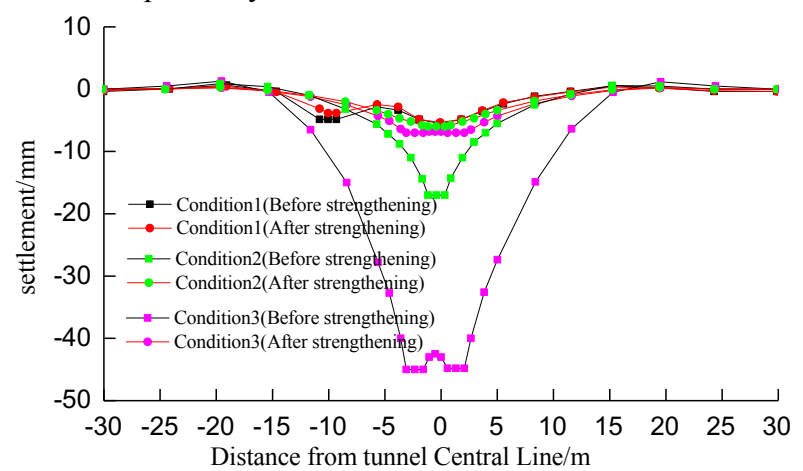

Fig.5. Horizontal surface curve of settlement changes, self-elaboration

The numerical results of plastic strain showed that the deformation of surrounding rock was under control, the settlement of the bridge foundation was small and the plastic zone without a trend after reinforcement measures were taken. To a certain extent, construction safety of the shield through the existing buildings is improved, although the extent cannot be quantitatively described. Therefore, a material average model is established by RFPA-2d with the help of the Weibull random distribution function to realize the material heterogeneity and random distribution of defects, simulated progressive failure of surrounding rock in the process of strength reduction, and calculated the overall safety factor.
The safety factor of tunnel shear failure is divided into two kinds [20]. One is the overall instability, which corresponds to the overall safety factor. The other is the tunnel's local instability, which generally occurs in joints and fissures in the rock mass and is relevant to the corresponding partial safety factors. In this paper, $F_{s}[6]$ is the overall safety coefficient, which is defined as: when the shear strength parameters of rock and soil body $\mathrm{c}$ and $\varphi$ reached critical values $c^{\prime}$ and $\varphi^{\prime}$, rock mass will be in a critical state of equilibrium, including:

$$
c^{\prime}=\frac{c}{F}
$$

Through the reduction factor and the current calculation step of instability, the strength reserve safety coefficient $F_{s}$ before the reduction of surrounding rock can be obtained:

$$
F_{s}=1 /(1-n \times \text { step })
$$

In the formula, $\mathrm{n}$ represents the reduction factor, which is 0.02 , and step is the current calculation step before the reduction instability destruction.

Fig. 6 is the shear stress diagram where different reduction steps occur. In Fig.6(1)(b), the reduction step is 25 - 36, where 25 is the total reduction steps, and 36 stands for the 25 th of the 36 steps, that is "step in step" , E can be calculated by the above elastic constitutive relation if the load cell damage according to the failure criterion. Thus, $\mathrm{E}$ can be used to reflect stress redistribution caused by the unit performance weakening and failure in the condition of invariable in the external load of recalculation, until a new unit damage can not be found in this step. The rock mass strength then continues to reduce and enters the next analysis step. 
Qian-cheng Fang, Li Shang, Yong-hui Shang, Ying Zhao and Ning-yi OU /

Journal of Engineering Science and Technology Review 9 (1) (2016) 131 - 138

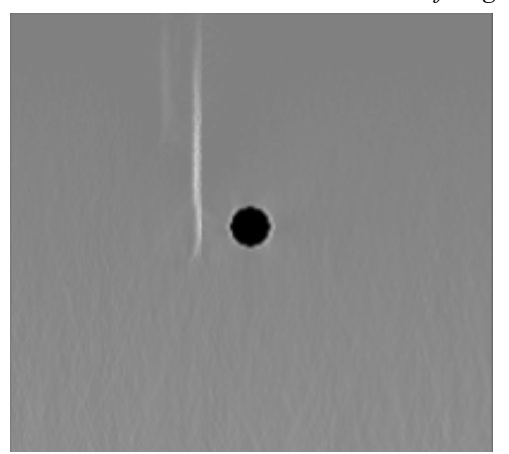

(a)Tunnel excavation
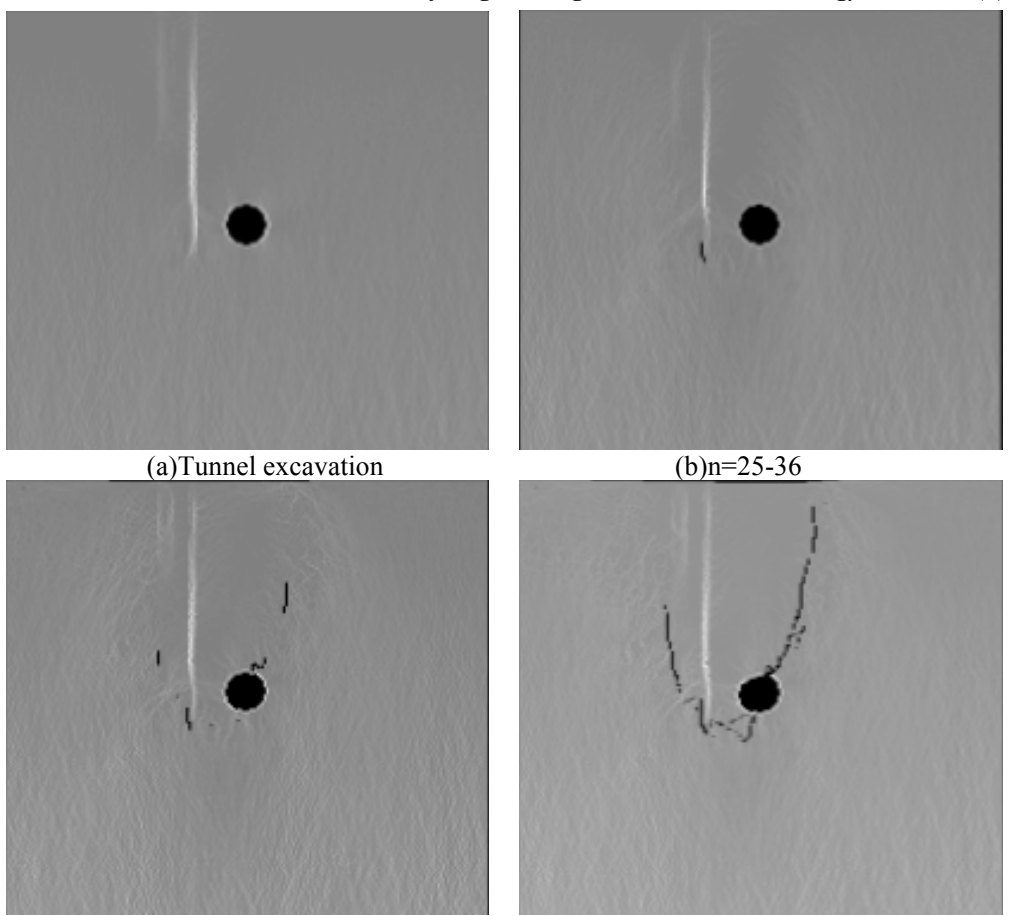

(d) $n=25-42$

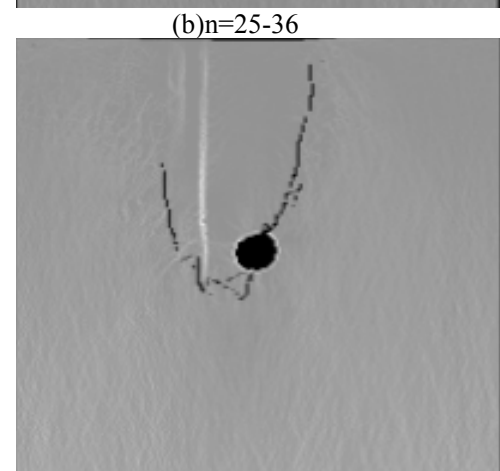

(e) $n=25-44$

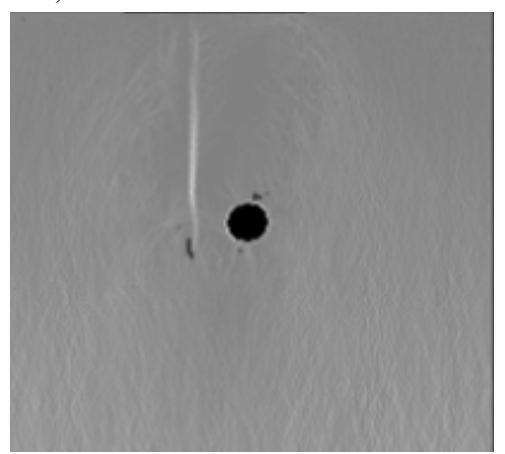

(b) $n=25-36$

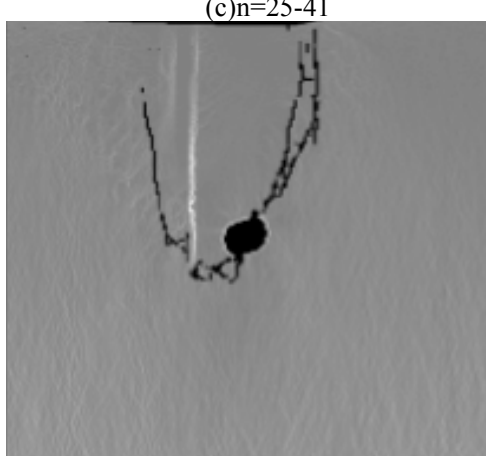

(f) $n=25-46$

(1)Condition 1: Different reduction step under shear stress(after strengthening)

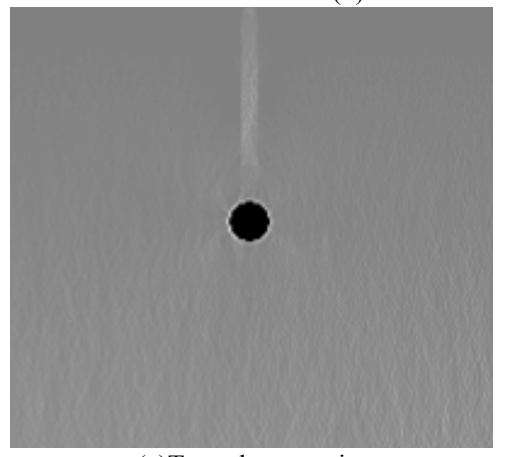

(a)Tunnel excavation
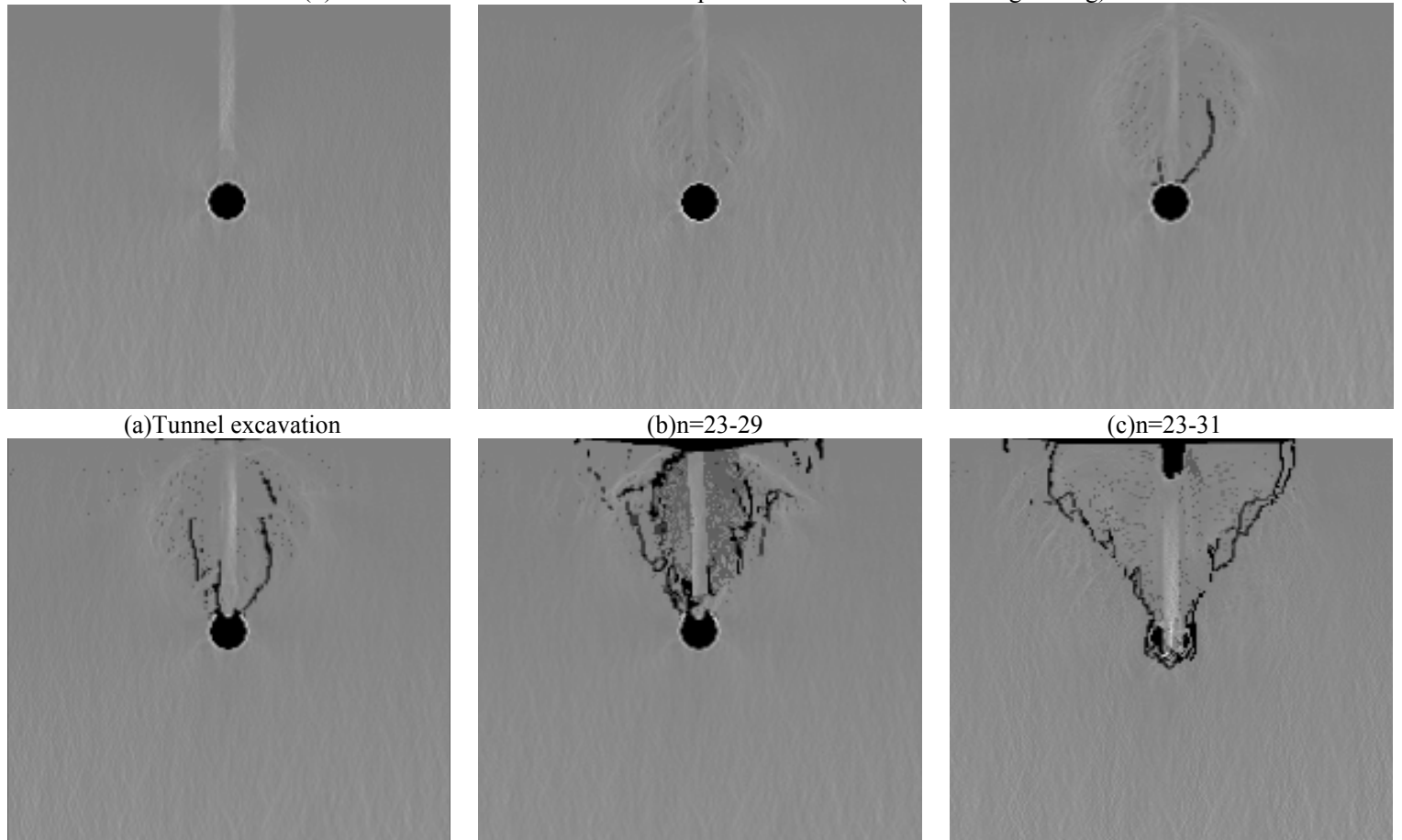

(d) $n=23-32$

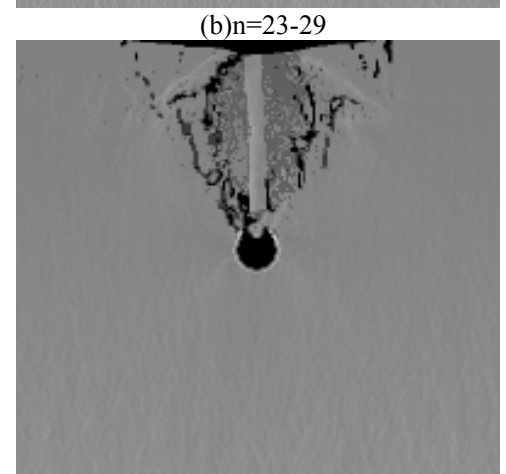

(e) $n=23-33$

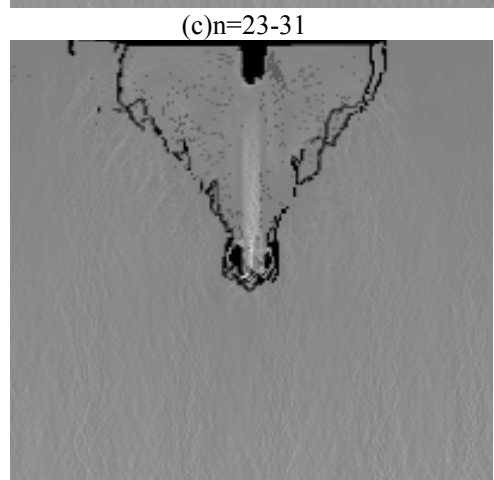

(2)Condition 2: Different reduction step under shear stress(after strengthening)

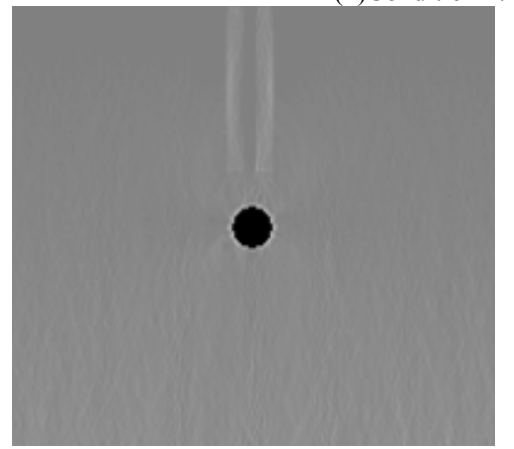

(a)Tunnel excavation

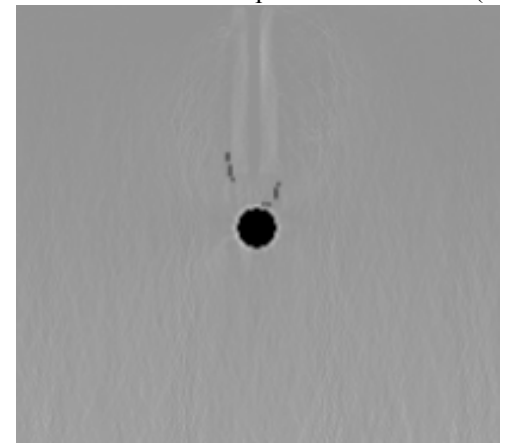

(b) $n=19-28$

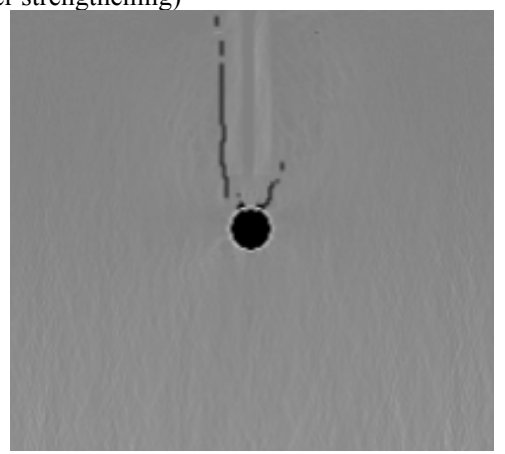

(c)n=19-31 

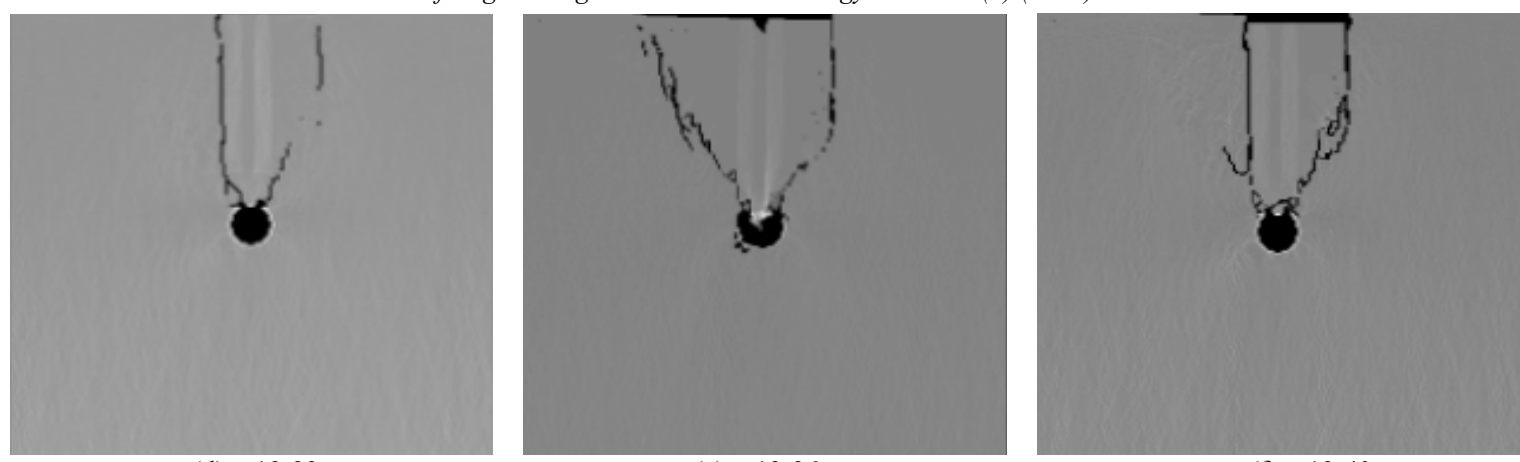

(3)Condition 3: Different reduction step under shear stress(after strengthening)

Fig.6. Shear stress in different reduction steps, self-elaboration

From Fig.6, it can be known that the cracks in the tunnel in working conditions $1 \sim 3$ are larger when the reduction step is $\mathrm{n}=25 \sim 36, \mathrm{n}=23 \sim 40$ and $\mathrm{n}=19 \sim 39$ respectively. Combined with Eq. 2, the safety factors of the corresponding conditions before and after reinforcement (Tab. 5) can be calculated. As can be seen from Table 5, the safety factors of working conditions $1 \sim 3$ were increased by $4.1 \%, 55.46 \%$ and $55.46 \%$ respectively after reinforcement.

Table 5. Safety coefficient statistics, self-elaboration

\begin{tabular}{ccc}
\hline Conditions & $\begin{array}{c}\text { Safety factor } \\
\text { (before reinforcement) }\end{array}$ & $\begin{array}{c}\text { Safety factor } \\
\text { (after reinforcement) }\end{array}$ \\
\hline Condition 1 & 1.92 & 2.00 \\
Condition 2 & 1.19 & 1.85 \\
Condition 3 & 1.14 & 1.61 \\
\hline
\end{tabular}

\subsection{Field Monitoring Test}

During the construction process, the survey points were arranged on the site to monitor existing buildings settlement, ground surface settlement, the surrounding rock convergence, vault displacement. From Figure 7, it can be seen that the value of the maximum settlement of the surface above the tunnel's surrounding rock convergence is less than $7 \mathrm{~mm}$, the surrounding rock convergence is less than $2 \mathrm{~mm}$, displacement is less than $3 \mathrm{~mm}$, and the bridge pile foundation settlement is less than $5 \mathrm{~mm}$ (a limit of $30 \mathrm{~mm}$ was given in the specification) after reinforcement.

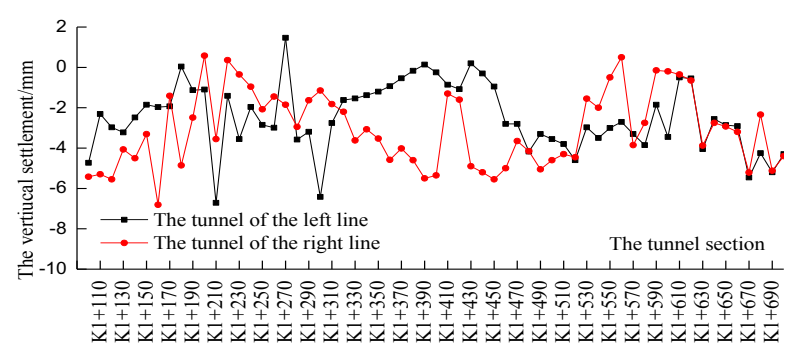

(1)The surface Vertical settlement above tunnel Central Line

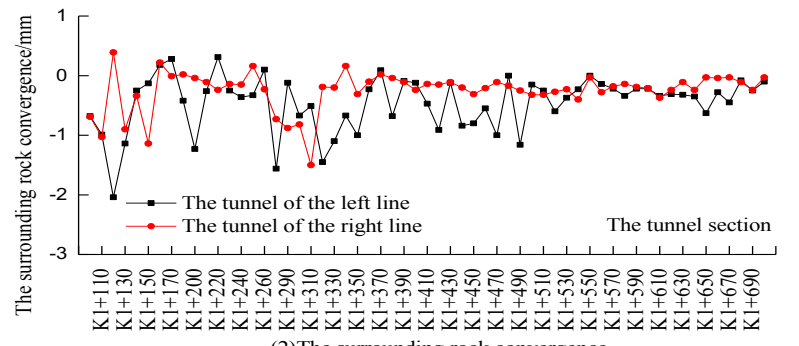

(2)The surrounding rock convergence
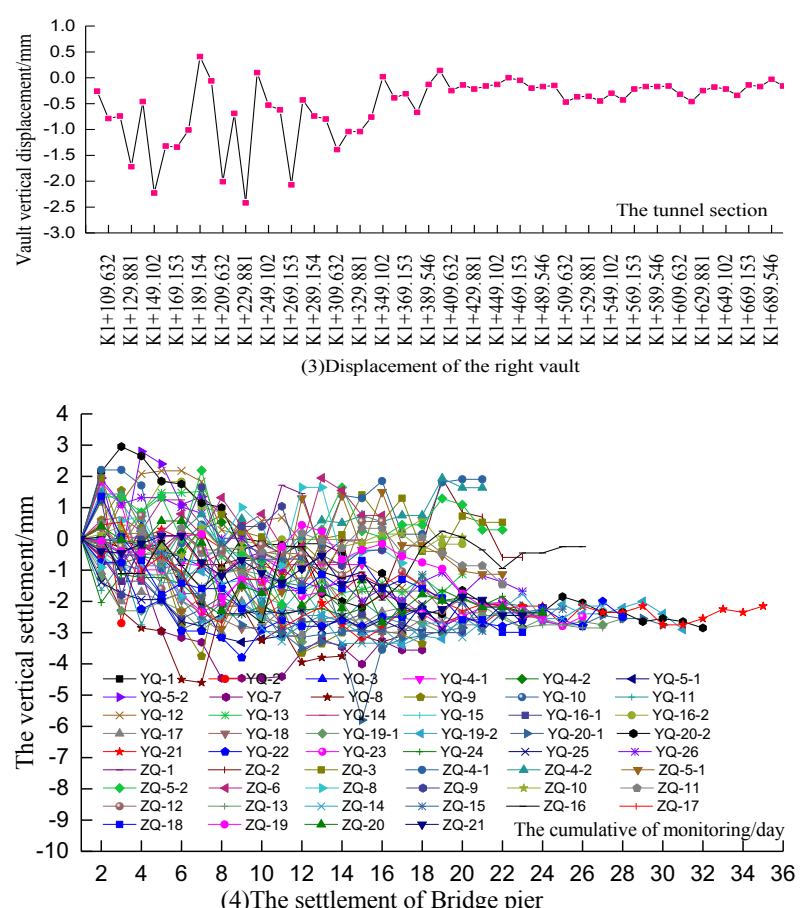

Fig.7. Field data, self-elaboration

According to the above analysis, the surrounding rock deformation and plastic zone area were reduced, the overall safety factor was increased, the settlement of the bridge foundation, the surrounding rock convergence, and the vault's displacement of surrounding rock were decreased, the bridge's normal use and tunnel's construction were ensured after the reinforcement measures are taken.

\section{Conclusions}

This paper firstly analyzed the reinforcement measures of the shield tunneling under overpass structure, then established two numerical models based on a detailed engineering example. The control effects of reinforcement were evaluated by numerical simulations, which was consistent with the monitoring data. The main conclusions are drawn as follows:

(1) Using the steel support method rather than load-bearing underpinning on the pile foundation, which is less than $2 \mathrm{~m}$ to the shield tunnel, can effectively avoid the damage to the original pier structure.

(2) From the results of numerical calculation, after reinforcement(conditions $1 \sim 3$ ), the maximum deformation values of the surrounding rock were reduced by $4.9 \%$, 
$12.2 \%$ and $48.46 \%$, and the maximum values of surface subsidence were reduced by $5.6 \%, 72.2 \%$ and $88.64 \%$, and the overall safety factors were increased by $4.1 \%, 55.46 \%$ and $55.46 \%$ respectively. The results of numerical calculation verified the necessity of the reinforcement measures.

(3) From the results of measured data, the maximum settlement value of the surface above the tunnel entrance was less than $7 \mathrm{~mm}$, convergence value of surrounding rock was less than $2 \mathrm{~mm}$, displacement of the vault was less than $3 \mathrm{~mm}$, and the settlement value of bridge foundation was less than $5 \mathrm{~mm}$ (the specification sets a limit of $30 \mathrm{~mm}$ ) after reinforcement. The measured datas verified the rationality of the reinforcement measures.

(4) Based on the experimental data and numerical simulations, the effect of reinforcement can be assessed from the aspects of surrounding rock deformation, plastic zone development and safety factor, which also provides reference for evaluating the similar projects.

\section{Acknowledgements}

This work was supported by the Key Scientific Research Projects of Colleges and Universities in Henan Province (16A120015).

\section{References}

1. Lu M L, Liu W N, Luo F R, et al. "Review on risk assessent methods for tunneling and underground projects", Journal of engineering geology, 14(04), 2006, pp. 462-468.

2. Ferrero A M, Segalini A \& Giani G P. "Stability analysis of historic underground quairies", Computers and Geotechnics, 37(4), 2010, pp.476-486.

3. Zhang Z W. "Settlement Control Technology of an Underground Tunnel Underneath the Existing Subway Loop", Municipal Engineering Technology, 29(6), 2011, pp.70-74.

4. He H J, Liu W N, et al. "Summary of Current Studies and Discussions on the Effects of Metro Construction on Adjacent Bridge Piles", Urban Rapid Rail Transit, 19(5), 2006, pp.85-88.

5. Liu B C. "Problems on geomechanics in need of immediate deep research in subway construction", Railway Construction Technology, 3, 2000, pp.1-3.

6. Li S, Feng X, Li Z, et al. "Evolution of fractures in the excavation damaged zone of a deeply buried tunnels during TBM construction", International Journal of Rock Mechanics and Mining Sciences, 55, 2012, pp. 125-138.

7. Hutchinson D J, Diederichs M, Pehme P, et al. "Geomechanics stability assessment of World War I military excavations at the Canadian National Vimy Memorial Site, France", International Journal of Rock Mechanics and Mining Sciences,45(1), 2008, pp. 59-77.

8. Wang M S. "An overview of development of railway, tunnels and underground works in china", Tunnels Construction, 30(4), 2010, pp. $351-364$.

9. Logangthan N, Oganathan N, et al. "Centrifuge model testing of tunneling-induced ground and pile deformations", Geotechnique, 50(3), 2000, pp. 283-294.

10. Li Y S, Huang H Y. "Computation of Mechanical Effects on Adjacent Piles by Shield Excavation", Journal of Tongji University (Natural Science), 25(3) 1997, pp. 274-280.
11. Ruan L W, Li Y S. "Study on deformation and stress of adjacent piles induced by shield construction", The Tunnel and Underground Engineering, 18(9), 1997, pp.18-23.

12. Wang Z S, Zhang D L. "Key Techniques on Shallow Embedded Tunnel Constructed Beneath Existing Subway Tunnel", Chinese Journal of Rock Mechanics and Engineering, 26(supp.2), 2007, pp. 4208-4214.

13. Burland J B, Standing J R \& Jardine F M. "Building Response to Tunneling-case Studies from Construction of the Jubilee Line Extension. Volume 1: Projects and Methods", Thomas Telford Publishing, England, London, 2001.

14. Ye S L, Wang Y J, Xu G Z, et al. "Technology of foundation underpinning”, Railway Publishing House, China, Beijing, 1991.

15. Wang W B, Liu W N, et al. "Discussion on risk management in urban subway projects", Journal of Beijing Jiaotong University, 35(4), 2011, pp. 33-37.

16. Zhai J Q, Jia J, Xie X L. "Practice of partition wall in the building protection projects deep excavation", Chinese Journal of Underground Space and Engineering, 6(1), 2010, pp. 162-166.

17. Zheng G, Du Y M, Diao Y. "Optimization Analsysis of Efficiency of Isolation Piles in Controlling the Deformation of Existing Tunnels Adjacent to Deep Excavation", Chinese Journal of Rock Mechanics and Engineering, 34(supp.1), 2015, pp. 3499-3509.

18. Zhu W C, Tang C A, Yang T H, et al. "Constitutive relationship of mesoscopic elements used in RFPA-2D and its validations", Chinese Journal of Rock Mechanics and Engineering, 22(1), 2003, pp. 24-29.

19. Lee K \& Rowe R K. "Finite element modeling of the threedimensional ground deformations due to tunneling in soft cohesive soils: Part I-method of analysis", CoBipnters and Geotechnics, 10(2), 1990, pp. 87-109.

20. Tang C A, Li L C, Li C W, et al. "RFPA strength reduction method for stability analysis of Geotechnical Engineering", Chinese Journal of Rock Mechanics and Engineering, 25(8), 2006, pp.15221530 . 